gration proceeds as in the previous case, a grid in the $(x, y)$ plane being chosen and interpolation among these grid points being used to advance the integration in time.

However, attempts at extending the integration method in an obvious way to general systems of hyperbolic equations in one space variable have not been successful. While the method presented here is marginally stable, the attempted generalizations have suffered from instabilities.

TABLE 1

Values of $u(x, t)$ at $t=2.4$.

\begin{tabular}{l|l|l|l|l|l}
\hline$x$ & \multirow{2}{*}{ Exact $u$} & \multicolumn{4}{|c}{ Computed $u$} \\
\cline { 2 - 6 } & & $\Delta=0.02$ & $\Delta=0.10$ & $\Delta=0.20$ & $\Delta=0.30$ \\
\hline-.48 & 0.004167 & 0.003713 & 0.003657 & 0.003584 & 0.003508 \\
-.40 & 0.0208 & 0.020805 & 0.02056 & 0.02024 & 0.01991 \\
-.32 & 0.0375 & 0.03738 & 0.03694 & 0.03637 & 0.03576 \\
-.24 & 0.0542 & 0.05399 & 0.05336 & 0.05253 & 0.05165 \\
-.16 & 0.0708 & 0.07063 & 0.06981 & 0.06869 & 0.06745 \\
-.08 & 0.0875 & 0.08748 & 0.08621 & 0.08484 & 0.08255 \\
.00 & 0.1000 & 0.10022 & 0.10034 & 0.10000 & 0.10072 \\
+.08 & 0.1000 & 0.09994 & 0.10000 & 0.10000 & 0.10002 \\
+.16 & 0.1000 & 0.10000 & 0.10000 & 0.10000 & 0.10000 \\
+.24 & 0.1000 & 0.10000 & 0.10000 & 0.10000 & 0.10000 \\
+.32 & 0.1000 & 0.10002 & 0.10000 & 0.10000 & 0.10000 \\
+.40 & 0.1000 & 0.10028 & 0.10008 & 0.10000 & 0.10009 \\
+.44 & 0.1000 & 0.09924 & 0.09952 & 0.10000 & 0.10111 \\
+.46 & 0.1000 & 0.09785 & 0.10351 & 0.09884 & 0.08966 \\
+.48 & 0.1000 & 0.12222 & 0.08834 & 0.06096 & 0.04563 \\
+.50 & - & 0.00000 & 0.00000 & 0.00000 & 0.00000 \\
+.52 & 0.0000 & 0.00000 & 0.00000 & 0.00000 & 0.00000 \\
+.54 & 0.0000 & 0.00000 & 0.00000 & 0.00000 & 0.00000 \\
+.56 & 0.0000 & 0.00000 & 0.00000 & 0.00000 & 0.00000 \\
\hline
\end{tabular}

\title{
ON THE EQUIDISTRIBUTION OF PSEUDO-RANDOM NUMBERS*
}

\section{By J. N. FRANKLIN (California Institute of Technology)}

1. Introduction. In the paper [1] J. Moshman discusses a method for generating pseudo-random numbers. As Moshman points out, his method is an adaptation to decimal computers of a process used on a binary computer by J. Todd and O. Taussky Todd [2]. Another adaptation to decimal computers was made by M. Juncosa [3]. The method is used widely for computations in nuclear physics. For example, the method is used by E. Leshan [4] to study the transport of neutrons.

In this method one chooses an initial number $x_{0}$ and a multiplier $N$. Each of these numbers is represented in a digital computer by a word with a fixed number of digits, say $s$ digits. The product $N x_{0}$ contains $2 s$ digits, of which the $s$ least significant digits

*Received May 9, 1957. 
are used to represent $x_{1}$. Now $x_{2}$ is represented by the least significant $s$ digits of the $2 s$-digit product, $N x_{1}$, and so on.

In a digital computer the number $x_{0}$ is rational, and the sequence $x_{0}, x_{1}, x_{2}, \cdots$ is periodic. In the present paper the sequences $x_{0}, x_{1}, x_{2}, \ldots$ are considered in which $x_{0}$ is irrational. By means of the ergodic theorem it is shown that almost all of these sequences are equidistributed.

2. Application of the ergodic theorem. Let us normalize the number $x_{0}$ by requiring

$$
0 \leq x_{0}<1 \text {. }
$$

We set $N$ equal to an integer $>1$. Then

$$
x_{n}=N x_{n-1}-\left[N x_{n-1}\right] \quad(n=1,2, \cdots),
$$

where $[N x]$ stands for the greatest integer $\leq N x$. In this notation the Todds, Juncosa, and Moshman choose, respectively,

$$
\begin{array}{ll}
x_{0}=2^{-3}, & N=5^{2 k+1} ; \\
x_{0}=10^{-3}, & N=3^{4 k+1} ; \\
x_{0}=10^{-\bullet}, & N=7^{4 k+1} ;
\end{array}
$$

where $s$ is the number of digits in a word of the computer, and $k$ is some positive integer.

One may consider more general sequences by fixing a number $\theta$ and setting

$$
x_{n}=N x_{n-1}+\theta-\left[N x_{n-1}+\theta\right] \quad(n=1,2, \cdots) .
$$

Without loss of generality, we suppose $0 \leq \theta<1$. When $\theta=0$, we have the definition (2).

The sequence $x_{0}, x_{1}, \cdots$ is said to be equidistributed if, for every fixed $a, b$ in the range $0 \leq a<b \leq 1$,

$$
\frac{1}{n} \sum_{\substack{a \leq x \leq b \\ k=0, \cdots, n-1}} 1 \rightarrow b-a \text { as } n \rightarrow \infty .
$$

When $N=1$ in (3), we have the case considered in the classical paper [5] by $\mathrm{H}$. Weyl, who showed that for every value of $x_{0}$ the sequence (3) where $N=1$, is equidistributed if and only if $\theta$ is irrational.

By Weyl's result the sequences (3) where $N=1$, are equidistributed for all $x_{0}$ and almost all $\theta$. If the integer $N$ is greater than 1, we shall show that the sequences (3) are equidistributed for all $\theta$ and almost all $x_{0}$.

Theorem. Let $f(x)$ be an arbitrary integrable function in the sense of Lebesgue. Let $0 \leq x_{0}<1$, and let a sequence $x_{0}, x_{1}, x_{2}, \cdots$ be formed according to (3), where $N$ is a fixed integer $>1$ and $\theta$ is a fixed number with $0 \leq \theta<1$. Then for almost all $x_{0}$

$$
\frac{1}{n} \sum_{k=0}^{n-1} f\left(x_{k}\right) \rightarrow \int_{0}^{1} f(x) d x \text { as } n \rightarrow \infty .
$$

The equidistribution (4) for almost all $x_{0}$ follows at once from the theorem if $f(x)$ is defined to be the characteristic function of the interval $a \leq x<b$, which takes the value 1 or 0 according as $x$ lies within or outside of the interval.

In order to prove the theorem we make use of a statement of the ergodic theorem due to F. Riesz [6]: 
"Let a measurable set $\Omega$ be given, of finite or infinite measure, the corresponding measure and integral being defined according to Lebesgue, or more generally, by means of a distribution of positive masses. That being the case, let us designate by $T$ a pointtransformation which is single-valued (but not necessarily one-to-one) from $\Omega$ onto itself; and let us suppose that $T$ conserves measure in the sense that, $E$ being a measurable set, $T E$ its transform, and $E^{\prime}$ the set of points $P$ whose images appear in $T E$, the sets $E^{\prime}$ and $T E$ have the same measure. Then, if $f_{1}(P)$ is an integrable function and $f_{k}(P)=f_{1}\left(T^{k-1} P\right)$, the arithmetic mean of the functions $f_{1}, f_{2}, \cdots, f_{n}$ converges almost everywhere, as $n \rightarrow \infty$, to an integrable function $\varphi(P)$ which is invariant (almost everywhere) under $T . "$

Riesz gives an elegant proof of this form of the ergodic theorem, which is more general than G. Birkhoff's original form [7] in that the transformation $T$ is not required to have a unique inverse. After completing the proof, Riesz writes without elaboration:

"Let us add finally that in the case where $\Omega$ is of finite measure, it follows by integration term by term (which is permitted in this case because of the uniform integrability of the terms)

$$
\int_{\Omega} \varphi(P)=\int_{\Omega} f_{1}(P) . ”
$$

A proof of this statement is written out at the end of this paper.

In our case the set $\Omega$ consists of the interval $0 \leq x<1$. The transformation $T$ is

$$
T x=N x+\theta-[N x+\theta] .
$$

Each point $x$ in $\Omega$ is the image under $T$ of exactly $N$ points:

$$
T \frac{k+x-\theta}{N}=x \begin{cases}k=0, \cdots, N-1 & \text { if } x \geq \theta, \\ k=1, \cdots, N & \text { if } x<\theta .\end{cases}
$$

To show that $T$ preserves measure, it is sufficient to show that

$$
\int_{0}^{1} f(T x) d x=\int_{0}^{1} f(x) d x
$$

for all integrable functions $f(x)$. In fact, if $f(x)$ is the characteristic function of a measurable set $T E$, then $f(T x)$ is the characteristic function of the set $E^{\prime}$ of all points whose images lie in $T E$, and Eq. (8) states that the sets $T E$ and $E^{\prime}$ have the same measure. By the periodicity of the function $x-[x]$, we have

$$
\begin{aligned}
\int_{0}^{1} f(T x) d x & =\int_{0}^{1} f(N x+\theta-[N x+\theta]) d x \\
& =\frac{1}{N} \int_{0}^{N} f(y+\theta-[y+\theta]) d y \\
& =\int_{0}^{1} f(y+\theta-[y+\theta]) d y \\
& =\int_{\theta}^{1+\theta} f(y-[y]) d y \\
& =\int_{0}^{1} f(y) d y,
\end{aligned}
$$

which establishes the required formula (8). Now we can prove our theorem. 
Proof of the theorem. By the definitions (3) and (6),

$$
x_{k}=T^{k} x_{0} \quad(k=0,1,2, \cdots) .
$$

By the ergodic theorem, there exists an integrable function $\varphi\left(x_{0}\right)$ defined almost everywhere for $0 \leq x_{0}<1$ such that

$$
\begin{gathered}
\frac{1}{n} \sum_{k=0}^{n-1} f\left(x_{k}\right) \rightarrow \varphi\left(x_{0}\right) \quad \text { a.e. as } n \rightarrow \infty, \\
\varphi\left(T x_{0}\right)=\varphi\left(x_{0}\right) \quad \text { a.e., }
\end{gathered}
$$

where "a.e." means "almost everywhere." To prove the theorem we must show that the function $\varphi\left(x_{0}\right)$ is almost everywhere equal to a constant, namely the average value of $f$ :

$$
\varphi\left(x_{0}\right)=\int_{0}^{1} f(x) d x \text { a.e. }
$$

Let the integrable function $\varphi(t)$ have the Fourier series

$$
\sum_{k=-\infty}^{\infty} c_{k} \exp (2 k \pi i t)
$$

According to the theorem of Fejer and Lebesgue [8], p. 415, this series is summable by arithmetic means to the sum $\varphi(t)$ for almost all $t$.

We shall show that

$$
c_{k}=\exp (-2 k \pi i \theta) c_{N k} \quad(k=0, \pm 1, \pm 2, \cdots) .
$$

By the invariance (11) of $\varphi$, we may write

$$
\begin{aligned}
c_{N k} & =\int_{0}^{1} \varphi(N t+\theta-[N t+\theta]) \exp (-2 N k \pi i t) d t \\
& =\frac{1}{N} \int_{0}^{N} \varphi(t+\theta-[t+\theta]) \exp (-2 k \pi i t) d t .
\end{aligned}
$$

By the periodicity of the exponential function,

$$
\begin{aligned}
\frac{1}{N} \int_{0}^{v} \varphi(t+\theta-[t+\theta]) \exp & (-2 k \pi i t) d t \\
= & \int_{0}^{1} \varphi(t+\theta-[t+\theta]) \exp (-2 k \pi i t) d t \\
= & \int_{0}^{1} \varphi(t) \exp [-2 k \pi i(t-\theta)] d t=\exp (2 k \pi i \theta) c_{k} .
\end{aligned}
$$

This establishes (14).

By iterating (14) $r$ times and taking absolute values, we find

$$
\left|c_{k}\right|=\left|c_{m}\right|, \quad \text { with } \quad m=N^{r} k \quad(r=1,2, \cdots) .
$$

But the Fourier coefficient $c_{m} \rightarrow 0$ as $m \rightarrow \pm \infty$. Keeping $k$ fixed in (17) and letting $r \rightarrow \infty$, we find

$$
c_{k}=0 \text { if } k \neq 0
$$


Therefore, the Fourier series (13) contains only the constant term $c_{0}$. Then the sum by arithmetic means equals $c_{0}$, and by the Fejerr-Lebesgue theorem

$$
\varphi(t)=c_{0} \quad \text { a.e. }
$$

It remains only to show that the constant $c_{0}$ has the value

$$
c_{0}=\int_{0}^{1} f(x) d x .
$$

This follows immediately from the statement $\int \varphi(P)=\int f_{1}(P)$, which is appended to the ergodic theorem. In our case, by (19),

$$
c_{0}=\int_{0}^{1} \varphi(t) d t=\int_{0}^{1} f(x) d x .
$$

This completes the proof of the theorem.

3. Proof that $\int \varphi(P)=\int f_{1}(P)$. As Riesz suggests, this statement, which is appended to his generalized ergodic theorem, may be proved by Lebesgue's convergence-theorem if the underlying space $\Omega$ has finite measure.

For any number $\lambda \geq 0$ we may define a bounded function $g_{i}(P)$ equal to $f_{1}(P)$ or to 0 according as $\left|f_{1}(P)\right| \leq \lambda$ or $\left|f_{1}(P)\right|>\lambda$. We may then define the remainder $h_{1}(P)=$ $f_{1}(P)-g_{1}(P)$. Let an arbitrary number $\epsilon>0$ be given. Since $f_{1}(P)$ is integrable, we may choose a number $\lambda=\lambda(\epsilon)$ so large that the corresponding function $h_{1}(P)$ satisfies the inequality

$$
\int_{\Omega}\left|h_{1}(P)\right|<\epsilon .
$$

We now apply the ergodic theorem separately to the functions $g_{1}(P)$ and $h_{1}(P)$, obtaining limits

$$
\begin{array}{r}
\lim _{n \rightarrow \infty} \frac{1}{n} \sum_{k=1}^{n} g_{k}(P)=\chi(P) \quad \text { a.e., } \\
\lim _{n \rightarrow \infty} \frac{1}{n} \sum_{k=1}^{n} h_{k}(P)=\psi(P) \quad \text { a.e. }
\end{array}
$$

Addition of these equations gives

$$
\varphi(P)=\chi(P)+\psi(P) \text { a.e. }
$$

Since every mean value appearing on the left-hand side of (23) has absolute value $\leq \lambda$ for all $P$, and since $m(\Omega)<\infty$, we may apply Lebesgue's theorem of bounded convergence to obtain

$$
\lim _{n \rightarrow \infty} \frac{1}{n} \sum_{1}^{n} \int_{\Omega} g_{k}(P)=\int_{\Omega} \chi(P) .
$$

Since $\int g_{k}(P)=\int g_{1}(P)$ for all $k,(26)$ gives

$$
\int g_{1}(P)=\int \chi(P) .
$$

Even though the function $h_{1}(P)$ is integrable, it is not obvious that all of the mean 
values

$$
\frac{1}{n} \sum_{1}^{n} h_{k}(P) \quad(n=1,2, \cdots)
$$

are majorized by a single integrable function $H(P)$. Therefore, to formula (24) we apply, not Lebesgue's theorem, but Fatou's theorem. Using the inequality (22), we find

$$
\begin{aligned}
\int|\psi(P)| & \leq \lim _{n \rightarrow \infty} \int\left|\frac{1}{n} \sum_{1}^{n} h_{k}(P)\right| \\
& \leq \lim _{n \rightarrow \infty} \frac{1}{n} \sum \int\left|h_{k}(P)\right|=\int\left|h_{1}(P)\right|<\epsilon .
\end{aligned}
$$

Combining our results, we find

$$
\begin{aligned}
\left|\int f_{1}(P)-\int \varphi(P)\right| & =\left|\int g_{1}(P)-\int \chi(P)+\int h_{1}(P)-\int \psi(P)\right| \\
& \leq\left|\int g_{1}(P)-\int \chi(P)\right|+\int\left|h_{1}(P)\right|+\int|\psi(P)| \\
& <0+\epsilon+\epsilon .
\end{aligned}
$$

Letting $\epsilon \rightarrow 0$, we obtain the required result $\int f_{1}(P)=\int \varphi(P)$.

\section{REFERENCES}

1. J. Moshman, The generation of pseudo-random numbers on a decimal calculator, J. Assoc. for Computing Machinery 1, No. 2, 88 (1954)

2. J. Todd, Some experiments on the Monte Carlo method, presented before the Institute of Mathematical Statistics, Boston, 1951

3. M. Juncosa, Random number generation on the BRL high-speed computing machines, Rep. No. 855, Ballistic Research Laboratories, Aberdeen Proving Ground, 1953

4. E. Leshan, A general purpose Monte Carlo program, American-Standard Atomic Energy Division Report, 1956

5. H. Weyl, Über die Gleichverteilung von Zahlen mod. Eins., Math. Ann. 77, 313 (1916)

6. F. Riesz, Sur la théorie ergodique, Commentarii Mathematici Helvetici 17, 221 (1945)

7. G. Birkhoff, Proof of the ergodic theorem, Proc. Natl. Acad. Sci. 18, 650 (1932)

8. E. Titchmarsh, The theory of functions, Oxford University Press, 2nd Ed., 1939

\section{SIMULTANEOUS INVARIANCE OF GENERALIZED SPHERICAL HARMONICS UNDER THE OPERATIONS OF TWO ROTATION GROUPS*}

\section{By R. N. D'HEEDENE (Cornell University)}

Abstract. A method is found for evaluating the coefficients of a sum of generalized spherical harmonics so that the sum will be simultaneously invariant under two rotation groups. The coefficients for a sum of ordinary spherical harmonics invariant under each individual group must first be known.

*Received May 15, 1957. 